\title{
BMJ Open Organ damage in sickle cell disease study (ORDISS): protocol for a longitudinal cohort study based in Ghana
}

Kofi A Anie, ${ }^{1,2}$ Vivian Paintsil, ${ }^{3}$ Ellis Owusu-Dabo, ${ }^{4,5}$ Daniel Ansong, ${ }^{3,6}$ Alex Osei-Akoto, ${ }^{3,6}$ Kwaku Ohene-Frempong, ${ }^{7}$ Kofi Aikins Amissah, ${ }^{3}$ Nicholas Addofoh, ${ }^{4}$ Ezekiel Bonwin Ackah, ${ }^{4}$ Amma Twumwa Owusu-Ansah, ${ }^{8}$ Solomon Fiifi Ofori-Acquah ${ }^{8,9}$

To cite: Anie KA, Paintsil V, Owusu-Dabo E, et al. Organ damage in sickle cell disease study (ORDISS): protocol for a longitudinal cohort study based in Ghana. BMJ Open 2017;7:e016727. doi:10.1136/ bmjopen-2017-016727

- Prepublication history for this paper is available online. To view these files please visit the journal online (http://dx.doi org/10.1136/bmjopen-2017016727).

Received 6 March 2017 Revised 1 June 2017 Accepted 3 July 2017
CrossMark

For numbered affiliations see end of article.

Correspondence to Kofi A Anie; kofi.anie@nhs.net

\section{ABSTRACT}

Introduction Sickle cell disease is highly prevalent in Africa with a significant public health burden. Nonetheless, morbidity and mortality in sickle cell disease that result from the progression of organ damage is not well understood. The Organ Damage in Sickle Cell Disease Study (ORDISS) is designed as a longitudinal cohort study to provide critical insight into cellular and molecular pathogenesis of chronic organ damage for the development of future innovative treatment.

Methods and analysis ORDISS aims to recruit children aged 0-15 years who attend the Kumasi Centre for Sickle Cell Disease based at the Komfo Anokye Teaching Hospital in Kumasi, Ghana. Consent is obtained to collect blood and urine samples from the children during specified clinic visits and hospitalisations for acute events, to identify candidate and genetic markers of specific organ dysfunction and end-organ damage, over a 3 year period. In addition, data concerning clinical history and complications associated with sickle cell disease are collected. Samples are stored in biorepositories and analysed at the Kumasi Centre for Collaborative Research in Tropical Medicine, Ghana and the Centre for Translational and International Haematology, University of Pittsburgh, USA. Appropriate statistical analyses will be performed on the data acquired.

Ethics and dissemination Research ethics approval was obtained at all participating sites. Results of the study will be submitted for publication in peer-reviewed journals, and the key findings presented at national and international conferences.

\section{INTRODUCTION}

Sickle cell disease (SCD) comprises a group of inherited red blood cell conditions that result from the abnormal production of haemoglobin. Over 305000 babies are born worldwide annually with SCD mostly in low and middle income countries, and about $75 \%$ or more of these births occur in sub-Saharan Africa, posing an increasing health burden $^{1}$ and contributing to early childhood

\section{Strengths and limitations of this study}

- The establishment of a longitudinal cohort study of children with sickle cell disease that intends to obtain biologic samples and clinical data to allow for future studies aimed at elucidating cellular and molecular pathogenesis of chronic organ damage.

- The prospective design will allow risk factors for organ dysfunction associated with sickle cell disease complications to be determined in a naturalistic study of children in a specialist Centre.

- Attrition or loss to follow-up of children with sickle cell disease after the initial study visit at the specialist Centre may lead to a bias and reduction in the internal validity of the study.

- This is a study in a single setting, and risk factors for organ damage characteristic of the particular environment and setting may not be generalizable to populations elsewhere. Further ecological studies will be required to examine risk factors for organ damage in multiple populations of children with sickle cell disease.

mortality. ${ }^{2}$ SCD affects approximately $2 \%$ of newborns in Ghana. ${ }^{3}$

Clinical syndromes of SCD include anaemia, infection, and the consequences of blood vessel blockage (vaso-occlusion). The latter deprives tissues of oxygen and is indicated as the cause of acute painful episodes, the hallmark of SCD, and other complications such as stroke, acute chest syndrome, priapism, leg ulceration and chronic organ failure. Stem cell transplantation offers curative possibilities although this is not universal, and other treatment options are generally limited in Africa. ${ }^{3}$ Improved knowledge and successful primary public health prevention strategies have positively impacted childhood survival transforming SCD into 
a chronic disease. Nonetheless, progressive deterioration of organ function and end-organ damage is inevitable and appears to be irreversible. ${ }^{4-6}$ The mechanisms that lead to these complications, studied mostly outside sub-Saharan Africa, are not fully understood. Further understanding through a longitudinal cohort study of patients with SCD may provide novel insights into cellular and molecular pathogenesis of chronic organ damage, and opportunities for the development of innovative treatment and precisely timed interventions to prevent onset of organ damage.

In Ghana, a pilot Newborn Screening (NBS) project for SCD was established in Kumasi (the second largest city) and Tikrom (a nearby rural community) from 1993 as an international collaborative study. ${ }^{7}$ Newborns identified with SCD are registered in the Kumasi Centre for Sickle Cell Disease (K-CSCD) at the Komfo Anokye Teaching Hospital (KATH), and followed up until 15 years of age through the Child Health Directorate. This NBS project was subsequently adopted by the Government of Ghana in 2010 to scale it up as a national public health programme.

All patients enrolled in the K-CSCD have their haemoglobin $(\mathrm{Hb})$ genotype confirmed with isoelectric focusing (IEF) in the neonatal period, and alkaline $\mathrm{Hb}$ electrophoresis beyond the neonatal period. K-CSCD provides comprehensive care for patients with available facilities and services including blood transfusion, radiology, laboratory, pharmacy, orthopaedics, and ophthalmology. There is a team of two consultant paediatricians, two specialist paediatricians, three residents (registrars), three house officers, a nurse in charge, eight other nursing staff, and three auxiliary personnel who help with data recording and retrieval of medical records on clinic days. Clinics are held every day.

\section{STUDY OBJECTIVES}

There are currently no data on the spectrum of organ dysfunction and end-organ damage in the SCD patient cohort attending K-CSCD. The Organ Damage in Sickle Cell Disease Study (ORDISS) was designed as a longitudinal cohort study of children with SCD attending K-CSCD to document acute events and the progressive deterioration in organ function with age, and to identify candidate and genetic markers of specific organ dysfunction and end-organ damage. Specific objectives are:

1. To determine the proportion of children with SCD attending K-CSCD who develop specific organ dysfunction.

2. To determine levels of biomarkers of organ dysfunction (heart, kidney, liver, lung, brain and skeletal muscle) from multiple candidate plasma and urine samples.

3. To determine haematologic and haemolytic markers in the recruited children attending clinic for routine evaluations or acute illness management.

4. To compare clinical evidence of organ dysfunction with biochemical and genetic markers.

\section{METHODS AND ANALYSES}

ORDISS is an international collaborative study conducted at three institutions: Department of Child Health, KATH/ Kwame Nkrumah University of Science and Technology (KNUST), Ghana; Kumasi Centre for Collaborative Research in Tropical Medicine (KCCR), Ghana; and Centre for Translational and International Haematology at the Heart, Lung, and Blood Vascular Medicine Institute (VMI), University of Pittsburgh, USA.

\section{Participants and recruitment}

A consecutive purposive sampling method of all individuals willing to participate is being employed to recruit and follow-up participants for the next 3 years in a longitudinal design.

\section{Eligibility criteria}

Eligible participants are families of children with SCD comprising all genotypes, confirmed with both IEF and alkaline electrophoresis with cellulose acetate membrane, who are registered at K-CSCD, aged 0 to 15 years and younger at recruitment, and receive outpatient or inpatient care at KATH. Patients not known to KATH and older than 12 months of age must be registered at K-CSCD for at least 12 months prior to becoming eligible for enrolment.

\section{Exclusion criteria}

Exclusions are children with SCD and co-morbid chronic conditions including malignancies, seizure disorders, and history or clinical signs and symptoms of HIV infection. In addition, patients who cannot be followed-up for a minimum of 12 months during the study, and families who decline informed consent or assent are excluded.

\section{Recruitment and enrolment procedures}

ORDISS was initially introduced to the families of children with SCD at the monthly Sickle Cell Disease Association meeting, a national support group for parents and patients with SCD, which is held at KATH premises.

\section{Enrolment (Entry) Visit}

Consecutive clinic attending families are opportunistically approached during routine clinic visits, and the study introduced to them prior to phlebotomy. Signed or thumb-printed informed consent is obtained from parents/caregivers and assent from children with SCD aged 7 years and over. Consenting families (participants) are enrolled into the study, and the child (subject) is assigned a unique study identification number that will be used as the subject identifier throughout the study. Participants' demographics, clinical information, and past medical history are recorded. These include the subject's age, gender, standing height, weight, head circumference, heart rate, respiratory rate, blood pressure, oxygen saturation (SpO2), and SCD complications relating to eyes, ears, head, nose, and mouth. Examinations of the throat, lymph nodes, chest with auscultation, heart with auscultation, abdomen, liver, spleen, genitalia, 
extremities, joints, and neurological examinations are performed by specialist paediatricians or residents, and recorded. In addition, data on parental ethnicity, religion, marital status, and educational level are collected. All information gathered is written in the subject's medical records, and entered into an electronic Case Report Form (e-CRF).

Using standard practice of phlebotomy, ${ }^{8}$ blood is collected from each subject into di-potassium ethylenediaminetetraacetic acid ( $\mathrm{K}_{0}$ EDTA) tube and serum separator (SS) tube with gel, each $3-4 \mathrm{~mL} ; 10-20 \mathrm{~mL}$ of midstream urine is also collected from each subject at specific visits. Blood and urine samples are collected from $8 \mathrm{am}$ to $12 \mathrm{pm}$ on the clinic day. Urine samples are collected from children aged 3 years and over using sterile urine containers, while infant urine collection bags are used for younger children. The latter urine samples are subsequently decanted into sterile urine containers. The blood in the $\mathrm{K}_{2}$ EDTA tube is inverted 8-10 times to ensure adequate mixing of the blood with the EDTA anticoagulant; the blood in the SS tube is allowed to adequately clot. ${ }^{8}$ The samples from each subject are duly labelled with the specific study identification number. The $\mathrm{K}_{2}$ EDTA-anticoagulated blood samples are sent, in a cryobox at room temperature, to the KATH Laboratory where aliquots are taken and immediately used for haematologic analyses; these include full blood count (FBC) with white blood cell (WBC) differential, performed electronically, and reticulocyte count, performed manually. ${ }^{9}$ The remainder of the blood sample and the urine sample are then placed on wet ice in a cold box and transported to KCCR for further processing, storage and analyses.

Demographic and clinical information, as well as FBC results, of the subjects are entered into a tablet adapted specifically for ORDISS with CommCare software, which allows creation and management of mobile applications through a website. The study database was developed at KCCR where the server is also held. Data are transmitted via a mobile phone network, subject to strength of connectivity, at the end of each clinic day from KATH to the KCCR server for cloud storage and management. This is subsequently extracted into Microsoft Excel spreadsheet format for statistical analyses.

\section{Interim (Follow-up) Visit}

On subsequent annual visits after recruitment (ie, interim visits) over 3 years, clinical procedures and data collection will be replicated, with emphasis on each subject's current ailments, episodes of acute illness not treated at KATH, episodes of enuresis, and current medications. In addition, educational performance is assessed and documented from preceding school-term reports to determine whether this is maintained during the study. Furthermore, blood and urine samples will be collected and identical procedures applied. Additionally, the process of data transmission with tablets via internet to KCCR will be repeated for each interim visit.

\section{Acute illness visit and hospitalisation}

During acute illness of subjects, blood and urine samples will be collected together with samples for acute illness blood tests requested by attending clinicians. These blood tests will also help to rule out illness due to other infections such as malaria, and allow comparison with steady-state laboratory values. Identical volumes of blood and urine will be collected from each subject during an acute illness; the samples will be processed using equivalent outlined procedures before these are transported on wet ice to KCCR.

\section{Biorepository sample collection and analyses}

At KCCR, the blood samples collected at each ORDISS visit are centrifuged for segregation of the major blood components. Each of plasma (from $\mathrm{K}_{2}$ EDTA tube) and serum (from SS tube) is harvested and aliquoted into two (2) tubes for storage. Buffy coat is then collected from each sample into single tubes. Genomic DNA is manually extracted from aspirated buffy coat samples using the QIAamp DNA blood mini kit (QIAGEN, \# 51106). DNA extracts are stored in double eluates/aliquots. Each sediment of red cells is stored in a single tube after washing three times with 1X phosphate buffered saline. A $4.5 \mathrm{~mL}$ single aliquot of each urine sample is also stored. All samples at each stage (ie, recruitment, interim and acute illness) of the study will be processed with a consistent approach at KCCR, and stored at $-80^{\circ} \mathrm{C}$.

A duplicate biorepository (ie, single aliquots) of DNA extracts, plasma and serum samples is maintained at the VMI. The samples are transferred carefully into intact stockings, and organised into bundles in the stockings; the mouth of each stocking is tied with a string and labelled with a sticker that bears the stocking number, sample type (ie, serum, plasma or DNA), and stage (visit) collected. The stockings are then placed in a tank containing liquid nitrogen $\left(\mathrm{at}-196^{\circ} \mathrm{C}\right)$, the tank stoppered tightly, labelled and shipped via air flight to VMI, observing all protocols. An electronic file in Microsoft Office Excel format showing the samples in the various stockings and stocking bundles being shipped are also sent electronically to VMI. The first shipment of duplicate biorepository to VMI has already been completed, and will subsequently take place once every year.

A sub-aliquot of each deposit of red cells will be used for haemolysate preparation. The haemolysates will be analysed to ascertain the haemoglobin ( $\mathrm{Hb}$ ) phenotype and determine the percentage of foetal $\mathrm{Hb}(\mathrm{HbF})$ of subjects at KCCR. DNA extracts will also be analysed for genetic markers of organ injury at VMI. Plasma, serum and urine samples will be batch assayed for chemical biomarkers of haemolysis, organ dysfunction and end organ damage both at KCCR and VMI. Assays will be performed using standardised validated enzyme-linked immunosorbent assays and colorimetric techniques. Laboratory investigations $^{10-27}$ are presented in the table 1 , and concise definitions of organ damage with diagnostic criteria ${ }^{28}$ are shown in table 2. 
Table 1 Organ Damage in Sickle Cell Disease Study (ORDISS): Laboratory Investigations

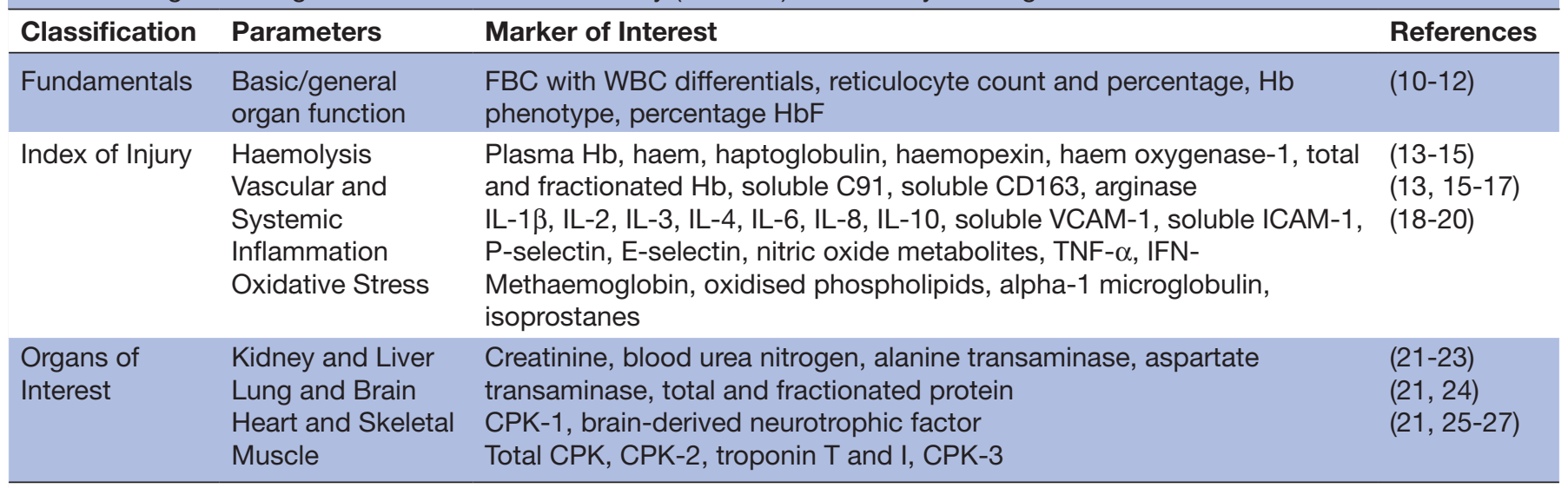

WBC=white blood cells, , VCAM=vascular cell adhesion molecule, , TNF=tumour necrosis factor.

CPK, creatine phosphokinase; FBC, full blood count; Hb, haemoglobin; ICAM, intravascular cell adhesion molecule; IL, interleukin; IFN, interferon; TNF, tumour necrosis factor; VCAM, vascular cell adhesion molecule, WBC, white blood cells.

\section{Statistical analysis}

Subjects will be allocated to the two extreme quartiles of biochemical and clinical evidence of organ dysfunction in a case-control design. The primary analysis will be ANOVA with either the actual measurements of biomarkers or those normalised by appropriate transformations. Genotypes will be the independent variable and the dependent outcomes will be biomarkers. Analyses will be run using the most recent version of the STATA software. In the event that there is evidence for a significant interaction between single nucleotide polymorphism (SNP) and a clinical event, analyses will also be run on organ damage and non-organ damage subjects independently. As with other phenotypes, it is likely that multiple genetic variants operate to affect the risk of specific clinical events more than any single SNP or plasma biomarker, independently. Thus, SNPs will be tested in 2-way-ANOVA and if a significant interaction term is observed, the effect size will be compared with the linear model. The results will be interpreted in light of known pathways and feedback loops for specific biomarkers. An exploratory analysis will be performed examining the relationship among biomarkers belonging to the same pathways. Specifically, we will interrogate data to see if correlations among these factors differ by the status of a clinical phenotype, which would suggest differences in the overall network of factors; this will be performed using Spearman's rank correlation and testing for heterogeneity among organ damage phenotypes using a t-test on the Fisher $r$-to-z transformations of the Spearman correlation coefficients performed. SNPs will be initially tested for association with the occurrence of acute organ damage as a dichotomous trait (eg, +ACS/-ACS). Statistical tests for differences in single locus allele and genotype frequencies will be calculated using PLINK. All loci will also be tested for Hardy-Weinberg equilibrium to assess the possibility of genotyping error. Genetic association will be concluded if the frequencies of either genotypes or alleles differ significantly between the extreme quartile classes $(\mathrm{p}<0.05)$. Odds ratios will be calculated using logistic regression.

\section{ETHICS AND DISSEMINATION}

\section{Ethical and safety considerations}

Research ethics approvals for ORDISS were obtained from both the Committee on Human Research, Publications and Ethics of KNUST (Approval No. CHRPE/ $\mathrm{AP} / 325 / 14$ ) and subsequently renewed approvals (No. CHRPE/AP/104/16 and No. CHRPE/140/17), and University of Pittsburgh Institutional Review Board (Approval No. PRO14010452). The study is currently in an active phase which commenced in May 2015, and just began year three. Informed consent (and assent where applicable) is obtained from all participants. Blood samples are routinely collected from children with SCD attending the K-CSCD at KATH and collection of urine samples is a non-invasive procedure. Data transmission from the K-CSCD at KATH to KCCR is secure. Biorepository samples are transported from KATH to KCCR and from KCCR to VMI, and stored appropriately according to international standards. Samples sent to VMI are de-identified, and there is an ethics (institution review board) approved material transfer agreement between the collaborating institutions.

\section{Dissemination}

The results of ORDISS will be submitted for publication in peer-reviewed journals, and the key findings presented at national and international sickle cell disease and haematology conferences.

\section{CONCLUSION}

It is envisaged that ORDISS will achieve its objectives and will substantially add to the modest amount of existing data on onset and progression of organ damage in children with SCD. ORDISS will also provide new insights into organ dysfunction and end-organ damage for appropriate and more precise timing of future therapeutic inventions. 


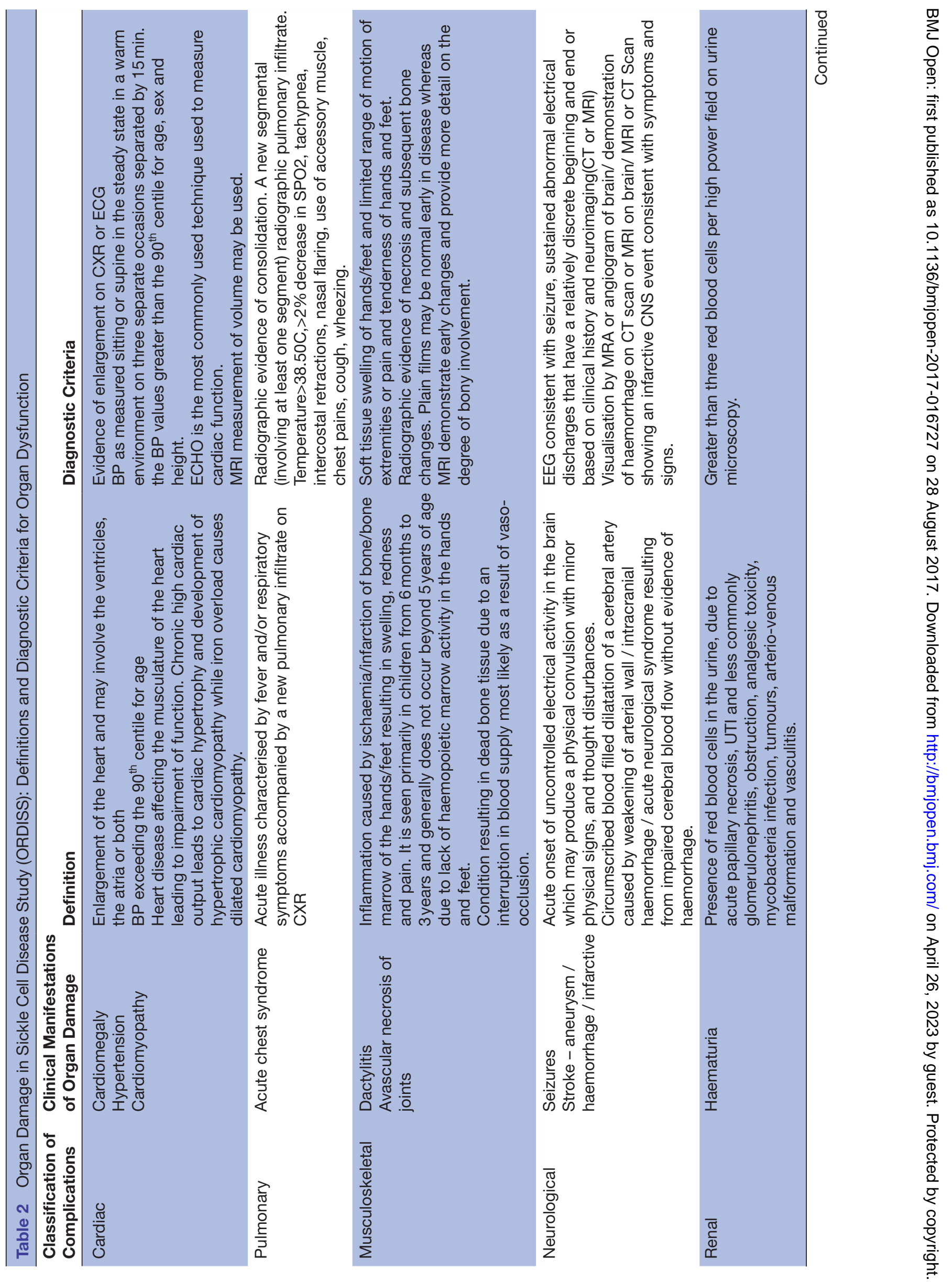


Author affiliations

${ }^{1}$ Haematology and Sickle Cell Centre, London North West Healthcare NHS Trust, Central Middlesex Hospital, London, UK

${ }^{2}$ Faculty of Medicine, Imperial College London, London, UK

${ }^{3}$ Directorate of Child Health, Komfo Anokye Teaching Hospital, Kumasi, Ghana ${ }^{4}$ Kumasi Centre for Collaborative Research in Tropical Medicine, Kwame Nkrumah University of Science and Technology, Kumasi, Ghana

${ }^{5}$ School of Public Health, Kwame Nkrumah University of Science and Technology, Kumasi, Ghana

${ }^{6}$ Department of Child Health, Kwame Nkrumah University of Science and Technology, Kumasi, Ghana

${ }^{7}$ Sickle Cell Foundation of Ghana, Kumasi, Ghana

${ }^{8}$ Center for Translational and International Hematology, Heart, Lung, and Blood Vascular Medicine Institute, University of Pittsburgh, Pittsburgh, Pennsylvania, USA ${ }^{9}$ School of Biomedical and Allied Health Sciences, University of Ghana, Accra, Ghana

Acknowledgements We are grateful to the participants of the study, and to all staff involved at Komfo Anokye Teaching Hospital, Kumasi Centre for Collaborative Research in Tropical Medicine and University of Pittsburgh.

Contributors SFO-A, KAA, EO-D, VP, DA, AO-A, KO-F made substantial contributions to the conception, and design of the study. KA, NA, EBA, ATO-A are involved in the acquisition or analyses of data. All the authors are accountable for all aspects of the work.

Funding ORDISS is supported with a collaborative seed grant from the Center for Translational and International Hematology, Vascular Medicine Institute, University of Pittsburgh, USA.

Competing interests None declared.

Patient consent None declared

Ethics approval Research Development Unit of Komfo Anokye Teaching Hospital, Committee on Human Research, Publications and Ethics of the Kwame Nkrumah University of Science and Technology and Komfo Anokye Teaching Hospital. University of Pittsburgh Institutional Review Board.

Provenance and peer review Not commissioned; externally peer reviewed. Data sharing statement None declared

Open Access This is an Open Access article distributed in accordance with the Creative Commons Attribution Non Commercial (CC BY-NC 4.0) license, which permits others to distribute, remix, adapt, build upon this work non-commercially, and license their derivative works on different terms, provided the original work is properly cited and the use is non-commercial. See: http://creativecommons.org/ licenses/by-nc/4.0/

(C) Article author(s) (or their employer(s) unless otherwise stated in the text of the article) 2017. All rights reserved. No commercial use is permitted unless otherwise expressly granted.

\section{REFERENCES}

1. Weatherall DJ. The challenge of haemoglobinopathies in resourcepoor countries. Br J Haematol 2011;154:736-44

2. Grosse SD, Odame I, Atrash HK, et al. Sickle cell disease in Africa: a neglected cause of early childhood mortality. Am J Prev Med 2011;41:S398-S405.

3. Ansong D, Akoto AO, Ocloo D, et al. Sickle cell disease: management options and challenges in developing countries. Mediterr J Hematol Infect Dis 2013;5:e2013062:2013062.

4. van Beers EJ, van Tuijn CF, Mac Gillavry MR, et al. Sickle cell disease-related organ damage occurs irrespective of pain rate: implications for clinical practice. Haematologica 2008;93:757-60.

5. Powars DR. Sickle cell Anemia and Major organ failure. Hemoglobin 1990;14:573-98.

6. Kato GJ, Gladwin MT, Steinberg MH. Deconstructing sickle cell disease: reappraisal of the role of hemolysis in the development of clinical subphenotypes. Blood Rev 2007;21:37-47.

7. Ohene-Frempong K, Oduro J, Tetteh $\mathrm{H}$, et al. Screening newborns for sickle cell disease in Ghana: table 1. Pediatrics 2008;121:S120 .2-S121.

8. World Health Organization.WHO guidelines on drawing blood: best practices in phlebotomy, 2010.

9. Bain BJ, Bates I, Laffan MA. Dacie and Lewis Practical Haematology. $12^{\text {th }}$ ed. London: Elsevier Health Sciences, 2016:27-30. 
10. Rees DC, Gibson JS. Biomarkers in sickle cell disease. Br J Haematol 2012;156:433-45.

11. Driscoll MC: sickle cell disease. Pediatrics in review 2007;28:259

12. Bender M. Seibel GD: sickle cell disease, 2014.

13. Rother RP, Bell L, Hillmen P, et al. The clinical sequelae of intravascular hemolysis and extracellular plasma hemoglobin: a novel mechanism of human disease. JAMA 2005;293:1653-62.

14. Kristiansen $M$, Graversen $\mathrm{JH}$, Jacobsen $\mathrm{C}$, et al. Identification of the haemoglobin scavenger receptor. Nature 2001;409:198-201.

15. Philippidis P, Mason J, Evans B, et al. Landis R: hemoglobin scavenger receptor CD163 mediates interleukin-10 release and heme oxygenase-1 synthesis. Circulation research 2004;94:119-26.

16. Pathare A, Al Kindi S, Alnaqdy AA, et al. Cytokine profile of sickle cell disease in Oman. Am J Hematol 2004;77:323-8.

17. Wagener F, Feldman $\mathrm{E}$, de Witte $\mathrm{T}$, et al. Heme induces the expression of adhesion molecules ICAM-1, VCAM-1 and E selectin in vascular endothelial cells (44197), 1997.

18. Akohoue SA, Shankar S, Milne GL, et al. Energy expenditure, inflammation, and oxidative stress in steady-state adolescents with sickle cell Anemia. Pediatr Res 2007;61:233-8.

19. Milne GL, Musiek ES. Morrow JD: f2-isoprostanes as markers of oxidative stress in vivo: an overview. Biomarkers 2005;10:10-23.

20. Klings ES, Christman BW, McClung J, et al. Increased F2 isoprostanes in the acute chest syndrome of sickle cell disease as a marker of oxidative stress. Am J Respir Crit Care Med 2001;164:1248-52

21. Jeter C, J. Hylin M, W. Hergenroeder G, et al. N Moore A: biomarkers of organ Injury. Recent Patents on Biomarkers 2014;4:98-109.

22. Arslan $G$, Gemici $A A$, Yirgin IK, et al. Liver trauma grading and biochemistry tests. Emerg Radiol 2013;20:379-84.

23. Schinstock CA, Semret MH, Wagner SJ, et al. Urinalysis is more specific and urinary neutrophil gelatinase-associated lipocalin is more sensitive for early detection of acute kidney injury. Nephrol Dial Transplant 2013;28:1175-85.

24. Fremont RD, Koyama T, Calfee CS, et al. Acute lung injury in patients with traumatic injuries: utility of a panel of biomarkers for diagnosis and pathogenesis. J Trauma 2010;68:1121-7.

25. McLean AS, Huang SJ, Salter M. Bench-to-bedside review: the value of cardiac biomarkers in the intensive care patient. Crit Care 2008;12:215.

26. Saenger AK. A tale of two biomarkers: the use of troponin and CKMB in contemporary practice. Clin Lab Sci 2010;23:134.

27. Guild CS, deShazo M, Geraci SA. Negative predictive value of cardiac troponin for predicting adverse cardiac events following blunt chest trauma. South Med J 2014;107:52-6

28. Ballas SK, Lieff S, Benjamin LJ, et al. Definitions of the phenotypic manifestations of sickle cell disease. Am J Hematol 2010;85:6-13. 